\title{
ON THE SIZE OF FINITE SIDON SEQUENCES
}

\author{
SHENG CHEN
}

(Communicated by William W. Adams)

\begin{abstract}
Let $h \geq 2$ be an integer. A set of positive integers $B$ is called a $B_{h}$-sequence, or a Sidon sequence of order $h$, if all sums $a_{1}+a_{2}+\cdots+a_{h}$, where $a_{i} \in B \quad(i=1,2, \ldots, h)$, are distinct up to rearrangements of the summands. Let $F_{h}(n)$ be the size of the maximum $B_{h}$-sequence contained in $\{1,2, \ldots, n\}$. We prove that
\end{abstract}

$$
F_{2 r-1}(n) \leq\left((r !)^{2} n\right)^{1 /(2 r-1)}+O\left(n^{1 /(4 r-2)}\right) .
$$

Let $h \geq 2$ be an integer. A set of positive integers $B$ is called a $B_{h}$-sequence if all sums $a_{1}+a_{2}+\cdots+a_{h}$, where $a_{i} \in B \quad(i=1,2, \ldots, h)$, are distinct up to rearrangements of the summands.

A $B_{h}$-sequence is also called a Sidon sequence of order $h$ [6]. We say that $B$ is a $B_{h}$-sequence for $Z /(n)$ if $B$ is a finite $B_{h}$-sequence and all sums are distinct modulo $n$.

Let $F_{h}(n)$ denote the size of maximum $B_{h}$-sequences contained in the set of integers $\{1,2, \ldots, n\}$ and $f_{h}(n)$ the size of maximum $B_{h}$-sequence for $Z /(n)$. Then it follows from a simple combinatorial argument that

$$
F_{h}(n) \leq(h \cdot h !)^{1 / h} n^{1 / h} \text { and } f_{h}(n) \leq(h !)^{1 / h} n^{1 / h} .
$$

Erdös and Turan [2] proved that $F_{2}(n)<\sqrt{n}+O\left(n^{1 / 4}\right)$. On the other hand, Bose and Chowla [1] showed that, for every $h \geq 2$, there exists a $B_{h}$-sequence $B$ for $Z /\left(m^{h}-1\right)$ with $|B|=m$, where $m$ is a prime power. This implies that

$$
F_{h}(n) \geq(1+o(1)) n^{1 / h} .
$$

Therefore, $F_{2}(n)=(1+o(1)) \sqrt{n}$.

Erdös conjectured that $F_{2}(n)=\sqrt{n}+O(1)$. For $h=3$, Lee [4] obtained that

$$
F_{3}(n) \leq\left(\left(1-\frac{1}{6 \log _{2}^{2} n}\right) 4 n\right)^{1 / 3} .
$$

For $h=4$, Lindström [5] proved that

$$
F_{4}(n) \leq(8 n)^{1 / 4}+O\left(n^{1 / 8}\right) .
$$

Received by the editors May 22, 1992 and, in revised form, September 11, 1992.

1991 Mathematics Subject Classification. Primary 11B83; Secondary 11B50, 05 B10.

Key words and phrases. Additive number theory, difference sets, $B_{h}$-sequence, Sidon sequences. 
When $h=2 r \quad(r \geq 1)$, Jia [3] showed that

$$
F_{2 r}(n) \leq\left((r !)^{2} r n\right)^{1 / 2 r}+O\left(n^{1 / 4 r}\right)
$$

and

$$
f_{2 r}(n) \leq\left((r !)^{2} n\right)^{1 / 2 r}+O\left(n^{1 / 4 r}\right) .
$$

In this paper, we shall obtain a similar upper bound for $F_{2 r-1}(n)$ and $f_{h}(n)$ as well.

Theorem 1. For all $r \geq 1$,

$$
F_{2 r-1}(n) \leq\left((r !)^{2} n\right)^{1 /(2 r-1)}+O\left(n^{1 /(4 r-2)}\right) .
$$

Theorem 2. For all $r \geq 1$,

$$
f_{2 r}(n) \leq\left((r !)^{2} n\right)^{1 / 2 r}+O(1)
$$

and

$$
f_{2 r-1}(n) \leq(r !(r-1) ! n)^{1 /(2 r-1)}+O(1) .
$$

First, some notation. Let $B$ be a $B_{2 r-1}$-sequence. Let $A=r B$ where $r B$ denotes the set of all sums of $r$ not necessarily distinct elements in $B$. We have

$$
t=|A|=\left(\begin{array}{c}
k+r-1 \\
r
\end{array}\right) \geq \frac{k^{r}}{r !} .
$$

Let

$$
V=\{(a, b) \mid a, b \in r B\}=V_{0} \cup V_{1},
$$

where $V_{1}=V \backslash V_{0}$ and $V_{0}$ consists of all elements $(a, b)$ such that $a=\sum_{i=1}^{r} a_{i}$ and $b=\sum_{i=1}^{r} b_{i}$ with $a_{i}, b_{j} \in B$ and $a_{j} \neq b_{j}$ for all $1 \leq i, j \leq r$.

Lemma. For any integer $d$, there are at most $k / r$ elements $(a, b)$ in $V_{0}$ such that $a-b=d$.

Proof. Let $\left(a_{i}, b_{i}\right)$ be elements in $V_{0}, i=1,2, \ldots, s$, such that $a_{i}-b_{i}=d$ for all $1 \leq i \leq s$. Suffice to show that, if $s>k / r$, at least two of the $\left(a_{i}, b_{i}\right)$ 's are the same.

Now assume $s>k / r$. Write $a_{i}=\sum_{j=1}^{r} a_{i j}$ and $b_{i}=\sum_{j=1}^{r} b_{i j}$ where $a_{i j}, b_{i j} \in B$. Since $|B|=k$ and $s r>k$, there are at least two distinct pairs $(i, j)$ and $\left(i^{\prime}, j^{\prime}\right) \quad\left(1 \leq i, i^{\prime} \leq s\right.$ and $\left.1 \leq j, j^{\prime} \leq r\right)$ such that $a_{i j}=a_{i^{\prime} j^{\prime}}$. By the definition of $V_{0}, i \neq i^{\prime}$. But then we have $a_{i}-a_{i j}-b_{i}=a_{i^{\prime}}-$ $a_{i^{\prime} j^{\prime}}-b_{i^{\prime}}$. Hence $a_{i}-a_{i j}+b_{i^{\prime}}=a_{i^{\prime}}-a_{i^{\prime} j^{\prime}}+b_{i}$. As $B$ is a $B_{2 r-1}$-sequence and $a_{i j}$ 's and $b_{i j}$ 's are all mutually distinct, we have $\left\{a_{i 1}, a_{i 2}, \ldots, a_{i r}\right\}=$ $\left\{a_{i^{\prime} 1}, a_{i^{\prime} 2}, \ldots, a_{i^{\prime} r}\right\}$ and $\left\{b_{i 1}, b_{i 2}, \ldots, b_{i r}\right\}=\left\{b_{i^{\prime} 1}, b_{i^{\prime} 2}, \ldots, b_{i^{\prime} r}\right\}$. Therefore, $\left(a_{i}, b_{i}\right)=\left(a_{i^{\prime}}, b_{i^{\prime}}\right)$. This completes the proof of the Lemma.

Proof of Theorem 1. Let $u=\left\lfloor n^{(4 r-3) /(4 r-2)}\right\rfloor$ and $I_{m}=[-u+m,-1+m]$, $m=1,2, \ldots, r n+u, C_{m}=I_{m} \cap B$, and $c_{m}=\left|C_{m}\right|$. Then

$$
(t u)^{2}=\left(\sum_{m=1}^{r n+u} c_{m}\right)^{2} \leq(r n+u) \sum_{m=1}^{r n+u} c_{m}^{2} .
$$


Note $c_{m}^{2}$ is the number of elements $(a, b) \in V$ such that $a, b \in C_{m}$. Hence $-u<a-b<u$.

For any integer $d,-u<d<u$, by the Lemma, there are at most $k / r$ elements $(a, b)$ in $V_{0}$ such that $a-b=d$. And each such pair is counted $u-|d|$ times toward the sum $\sum_{m=1}^{r n+u} c_{m}^{2}$. Hence, as $\left|V_{1}\right| \leq O\left(k^{2 r-1}\right)$ and $k \leq$ $O\left(n^{1 /(2 r-1)}\right)$,

$$
\begin{aligned}
(t u)^{2} & \leq(r n+u)\left(\sum_{-u<d<u} \frac{k}{r}(u-|d|)+O\left(k^{2 r-1}\right)\right) \\
& =(r n+u)\left(\frac{k}{r} u^{2}+O\left(k^{2 r-1}\right)\right) .
\end{aligned}
$$

So,

$$
t^{2} \leq n k\left(1+O\left(n^{-1 /(4 r-2)}\right)\right)
$$

Hence,

$$
k \leq\left((r !)^{2} n\left(1+O\left(n^{-1 /(4 r-2)}\right)\right)\right)^{1 /(2 r-1)} \leq\left((r !)^{2} n\right)^{1 /(2 r-1)}+O\left(n^{1 /(4 r-2)}\right) .
$$

This proves Theorem 1.

Proof of Theorem 2. In the case $h=2 r-1$, we use the same settings $A$ and $V$. Since, for any $d \in Z /(n)$, there exist at most $k / r$ elements $(a, b) \in V_{0}$ such that $a-b=d$, we have

$$
t^{2}=|V|=\left|V_{0}\right|+\left|V_{1}\right| \leq \frac{k}{r} n+O\left(k^{2 r-1}\right) .
$$

Hence,

$$
\frac{k^{2 r}}{(r !)^{2}} \leq \frac{k}{r} n+O\left(k^{2 r-1}\right)=\frac{k}{r} n\left(1+O\left(n^{-1 /(2 r-1)}\right)\right)
$$

and

$$
\begin{aligned}
k & \leq(r !(r-1) ! n)^{1 /(2 r-1)}\left(1+O\left(n^{-1 /(2 r-1)}\right)\right)^{1 /(2 r-1)} \\
& =(r !(r-1) ! n)^{1 /(2 r-1)}+O(1) .
\end{aligned}
$$

This shows that $f_{2 r-1}(n) \leq(r !(r-1) ! n)^{1 /(2 r-1)}+O(1)$. Similarly, we have $f_{2 r}(n) \leq\left((r !)^{2} n\right)^{1 /(2 r)}+O(1)$, which completes the proof of Theorem 2 .

\section{ACKNOWLEDGMENT}

The author would like to thank W. Gu and X.-D. Jia for their valuable and intriguing discussion.

\section{REFERENCES}

1. R. C. Bose and S. Chowla, Theorems in the additive theory of numbers, Comment. Math. Helv. 37 (1962/1963), 141-147.

2. P. Erdős and P. Turan, On a problem in additive number theory and some related problems, J. Number Theory 38 (1941), 191-205.

3. X.-D. Jia, On finite Sidon sequences, J. Number Theory 44 (1993), 84-92. 
4. M. A. Lee, On $B_{3}$ sequences, Acta Math. Sinica 34 (1991), 67-71.

5. B. Lindström, $A$ remark on $B_{4}$-sequences, J. Combin. Theory 7 (1969), 276-277.

6. S. Sidon, Ein Satz uber trigonomietrische Polynome und seine Anwendun gen in der Theorie der Fourier-Reihen, Math. Ann. 106 (1932), 536-539.

Department of Mathematics, Southwest Texas State University, San Marcos, Texas 78666

E-mail address: sc03@swtexas.bitnet 